\title{
Aggressive presentation of breast implant-associated ALK-1 negative anaplastic large cell lymphoma with bilateral axillary lymph node involvement
}

\author{
BACHIR ALOBEID ${ }^{1}$, DEBORAH W. SEVILLA ${ }^{1}$, MAHMOUD B. EL-TAMER ${ }^{2}$, \\ VUNDAVALLI V. MURTY ${ }^{1}$, DAVID G. SAVAGE ${ }^{3}$, \& GOVIND BHAGAT ${ }^{1}$ \\ ${ }^{1}$ Department of Pathology, Columbia University, New York, USA, ${ }^{2}$ Department of Surgery, Columbia University, New York, \\ USA, and ${ }^{3}$ Division of Hematology and Medical Oncology, Department of Medicine, Columbia University, New York, USA
}

(Received 18 November 2008; revised 5 fanuary 2009; accepted 1 February 2009)

We recently encountered a unique case of breast silicone implant-associated ALK-1 negative anaplastic large cell lymphoma (ALCL) in a female with a remote history of breast carcinoma, which presented as a sinusoidal infiltrate in an axillary lymph node (LN) and had a highly complex karyotype.

A 68-year-old female had undergone right breast lumpectomy followed by right modified radical mastectomy with axillary LN dissection in 1991 for infiltrating moderately differentiated ductal carcinoma. No lymphovascular invasion or $\mathrm{LN}$ involvement was identified. She received six cycles of chemotherapy and 5 years of tamoxifen. In January of 1992, the patient underwent right breast reconstruction with a silicone implant. Sixteen years later the patient developed right axillary lymphadenopathy. Her past medical history was significant only for the prior breast carcinoma and biliary cirrhosis. She had no history of non-Hodgkin or Hodgkin lymphoma. Right axillary LN biopsy showed a large cell neoplasm infiltrating and expanding the sinuses (Figure1A). An exhaustive staining panel led to the diagnosis of an ALK-1 negative ALCL (Figure1B). A staging bone marrow biopsy showed no evidence of lymphoma.

A subsequent FDG-PET/CT scan showed a hypermetabolic focus (maximum SUV 7.4) posterior to the breast implant. The grossly intact implant was removed with capsulectomy. Histologic review of the fibrotic capsular tissue showed an infiltrate of large neoplastic cells as seen previously (Figure 2A), which had the following phenotype (Figure 2B): CD45-/+, CD30+, CD15+, EMA+, MUM1+, $\mathrm{CD} 2+$ and $\mathrm{CD} 4+$. These cells did not express CD3, CD5, CD7, CD8, ALK-1, CD56, CD20, PAX5, CD79a, OCT2, CD138, p53, or cytotoxic T-cell granule constituents (TIA-1, granzyme-B and perforin). In situ hybridisation for EBV mRNA was negative. The proliferation rate, as assessed with a stain for $\mathrm{Ki}-67$, was $>90 \%$. Cytogenetic analysis revealed highly complex clonal chromosome abnormalities, penta-ploidy range metaphases and homogenously staining regions (hsr's): $116-123,<5 \mathrm{~N}>, \mathrm{XX},-1$, $\operatorname{add}(1)(\mathrm{p} 36.3), \mathrm{i}(1)(\mathrm{q} 10), \mathrm{hsr}(1)(\mathrm{q} 21 \mathrm{q} 25),+2,+3 \times 2$, $+6, \operatorname{hsr}(7)(\mathrm{q} 32 \mathrm{q} 35) \times 2, \mathrm{i}(8)(\mathrm{q} 10),+9,+10, \operatorname{inv}(11)$ $(\mathrm{p} 15.1 \mathrm{q} 22.1) \times 3, \operatorname{add}(12)(\mathrm{q} 24.1),-13,-14,-15, \mathrm{i}$ (17)(q10), +19,-20,+1 8mar[cp13]/46, XX, inv(11) (p15.1q22.1)[7]. FISH analysis using T-cell receptor alpha/delta break-apart, ALK break-apart and D7S486/CEP7 probes showed no evidence of rearrangement or deletion. PCR analysis showed clonal T-cell receptor beta gene rearrangement.

Four months after initial diagnosis, the patient developed left-sided (contralateral) axillary lymphadenopathy, and a LN biopsy again disclosed sinusoidal infiltrates of ALCL. After completion of six 


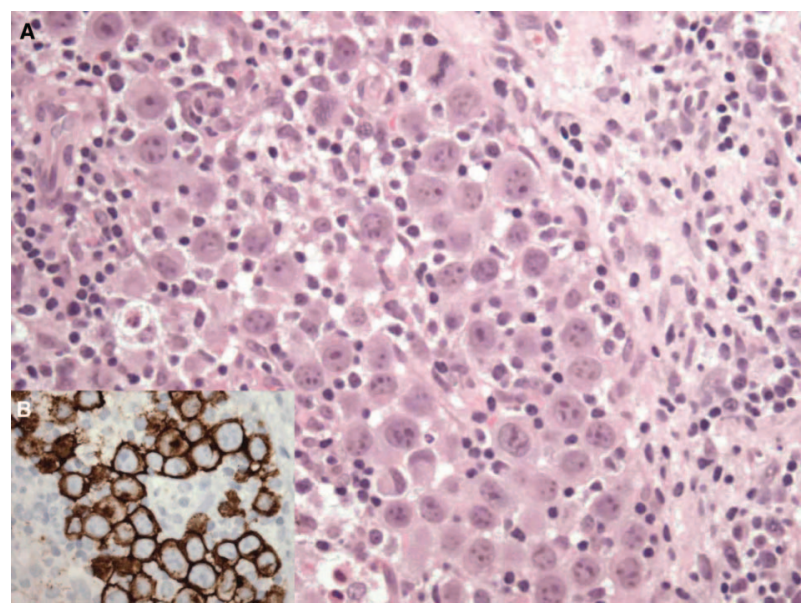

Figure 1. A: Lymph node biopsy showing anaplastic large cells within sinusoids $(\mathrm{H} \& \mathrm{E}, \times 200)$, (B) the cells are CD30 positive $(\times 400)$.

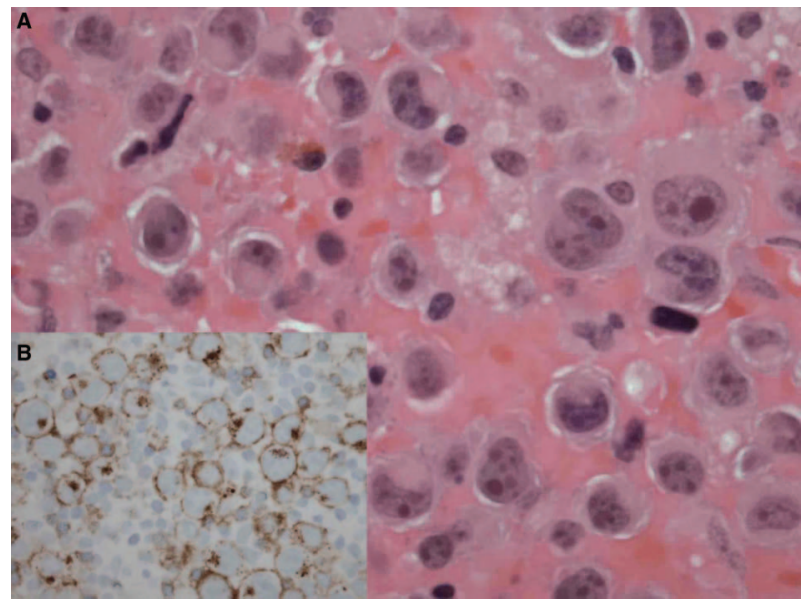

Figure 2. A: Anaplastic cells infiltrating the fibrous capsule (H\&E, $\times 400)$, (B) the cells express CD4 $(\times 400)$.

cycles of CHOP (cyclophosphamide, vincristine, doxorubicin, and prednisone) therapy, a repeat PET/CT scan showed a mildly hypermetabolic focus in the dome of the liver (SUV 2.8) and 2 minimally hypermetabolic foci in the left breast (SUV 0.7), suspicious for residual disease. Of note, she had also been under treatment for primary biliary cirrhosis and these lesions were not biopsied, therefore, it is unclear if the imaging findings represented lymphoma or a reactive condition.

Our case represents an ALK-1 negative ALCL arising in a breast capsule, in association with a silicone implant and bilateral axillary LN involvement. This case thus adds to the growing literature of breast prosthesis-associated lymphomas, with 17 cases published thus far [1-3], as well as a series of six unpublished cases [4].
A recent case report by Wong et al. and the seven other ALK-1 negative ALCL cases reviewed in their article presented in the breast without nodal involvement [2]. Only one patient had a relapse, while all others were living free of disease. The four cases reported by Roden et al. were also ALK-1 negative ALCL, which were described as 'therapy-resistant seroma' adjacent to saline or silicone implants; neoplastic cells being identified only in the serous fluid without tissue invasion [1]. Three of their patients were staged and showed no evidence of systemic disease. Three of the patients were alive without evidence of disease after a mean follow-up of 13 months (range of 9-20 months), suggesting that these lymphomas represent indolent T-cell lymphoproliferative disorders. Popplewell et al. described six cases of primary ALCL, including two ALK-1+ ALCL of the breast occurring in patients 5-20 years after implantation of silicone breast prostheses. All of their patients had lymphoma confined to the breast at the time of diagnosis; however, 2 suffered recurrences following initial therapy [4]. The latter underwent autologous stem cell transplantation, and showed no evidence of disease after a 2-year follow-up. A recent study by de Jong et al. described five additional cases of ALK-1 negative ALCL with 'dominant breast involvement' occurring in the fibrous capsules of saline breast implants placed 1-23 years before diagnosis [3]. Three of their cases had either bilateral breast involvement or nodal involvement at time of diagnosis and seemed to pursue a more aggressive clinical course, similar to our case. However, it is unclear if all their cases represented true primary breast lymphomas.

Although most lymphomas arising in the breast are of B-cell origin, the majority of those described in association with breast prostheses have a T-cell phenotype, and ALCL is the most common type described. The neoplastic lymphocytes in all published studies were negative for ALK-1 expression [1-3].

Interestingly, in our case, the lymphoma presented initially in a right axillary $\mathrm{LN}$ with a sinusoidal pattern of infiltration by large undifferentiated malignant cells, mimicking metastatic breast carcinoma. This represented a diagnostic challenge, especially given the remote history of breast carcinoma. The expression of CD15 was also unusual for ALCL. In addition, the clinical behaviour of our case was more aggressive than most cases described previously.

None of the previously published implantassociated ALK-1 negative ALCL have described any cytogenetic or karyotypic aberrations. The 
chromosomal regions altered in our case differ from those reported in a recent study of systemic ALK-1 negative ALCL [5]. Although cytogenetic aberrations of chromosome 1, 6 and 13 were noted in our case, the affected loci differed from those previously reported.

Despite the reported cases of implant associated malignancies and the intriguing overrepresentation of ALK-1 negative ALCL, the incidence of these types of lymphomas remains extremely low given that breast prostheses have been widely used for decades. However, oncologists and pathologists should be aware of this association and consider ALCL in their differential diagnosis when examining tissue removed during capsulectomy for failed or replacement breast prostheses, or when review of axillary LNs shows sinusoidal infiltrates of large cells, even if the patient had a prior history of carcinoma.

\section{References}

1. Roden AC, Macon WR, Keeney GL, Myers JL, Feldman AL, Dogan A. Seroma-associated primary anaplastic large-cell lymphoma adjacent to breast implants: an indolent T-cell lymphoproliferative disorder. Mod Pathol 2008;214:455-463.

2. Wong AK, Lopategui J, Clancy S, Kulber D, Bose S. Anaplastic large cell lymphoma associated with a breast implant capsule: a case report and review of the literature. Am J Surg Pathol 2008;32:1265-1268.

3. de Jong D, Vasmel WLE, de Boer JP, Verhave G, Barbe E, Casparie MK, et al. Anaplastic large-cell lymphoma in women with breast implants. J Am Med Assoc 2008;300: 2030-2035.

4. Popplewell L, Chang K, Olevsky O, Nademanee A, Forman S. Primary anaplastic large cell lymphoma of the breast occurring in patients with silicone breast implants. Blood 2004;104 American Society of Hematology:Abstract 4563.

5. Zetti A, Rüdiger T, Konrad MA, Chott A, Simonitsch-Klupp I, Sonnen R, et al. Genomic Profiling of peripheral T-cell lymphoma, unspecified, and anaplastic large T-cell lymphoma delineates novel recurrent chromosomal alterations. Am J Pathol 2004;164:1837-1848. 